\title{
Association between sedentary behaviour inside and outside preschools and executive function in preschoolers
}

Thayna Bezerra ( $\square$ thaynaalves.ef@gmail.com )

Universidade Federal da Paraiba Centro de Ciencias da Saude https://orcid.org/0000-0003-3296-4747 Irene Esteban-Cornejo

University of Granada: Universidad de Granada

Natalia Batista Albuquerque Goulart

Federal University of Paraiba: Universidade Federal da Paraiba Centro de Ciencias da Saude Jorge Augusto Pinto Silva Mota

University of Porto: Universidade Do Porto

Anastácio Neco Souza Filho

Federal University of Paraiba: Universidade Federal da Paraiba Centro de Ciencias da Saude

Paulo Felipe Ribeiro Bandeira

Regional University of Cariri: Universidade Regional do Cariri

Clarice Maria Martins

Federal University of Paraiba: Universidade Federal da Paraiba Centro de Ciencias da Saude

\section{Research Article}

Keywords: Sedentary Behaviour, Efficiency Inverse, Executive Function, Preschoolers.

Posted Date: April 16th, 2021

DOl: https://doi.org/10.21203/rs.3.rs-401898/v1

License: (c) (i) This work is licensed under a Creative Commons Attribution 4.0 International License. Read Full License 


\section{Abstract}

Preschool children spend a large part of their day at school, and a large part of that time they spend in sedentary behaviour (SB). Although SB negatively affects regions of the brain responsible for cognition, it is believed that the type of SB performed can favour executive functions' performance. The present study aimed to analyse the association between SB inside and outside preschools and EF tasks in preschool children. Thus, 73 pre-school children (60.3\% girls) were evaluated, with a mean age of $55.0 \pm 9.1$ months. The SB and physical activity (PA) were objectively assessed using accelerometers. The executive function (EF) was assessed using the Go / No Go paradigm by Early Years Toolbox - YET, and for the purposes of analysis, the inverse efficiency of Go and No Go accuracy were considered. Body Mass Index (BMI) was calculated [weight/height ${ }^{2}\left(\mathrm{~kg} / \mathrm{m}^{2}\right)$ ]. The preschool secretary provided the children's sex and age. To assess possible associations between $\mathrm{EF}$ and $\mathrm{SB}$, with sex, age, BMI and PA as covariates, a structural equation model, using multivariate linear regression analysis, was conducted. A significant and positive association between SB in weekend days and IE of Go $(b=0.61 ; p<0.001)$ was observed. The model's general explaining $51.9 \%$ of IE, and $2 \%$ of Ac-No Go.

Conclusion: the model presented in this study showed that SB on weekend days showed a significant and adverse association with children's IE, suggesting that excessive SB on these days may be harmful to EF in early child.

\section{Introduction}

Excessive time spent in sedentary behaviour (SB) has been characterized as a health determinant, due to its negative influence on cardiometabolic markers and its positive association with all-cause mortality in adult population [1]. Prior researches have also shown the negative effect of SB on brain structure and cognitive skills, such as executive function (EF), in childhood and adolescence [2, 3].

EF corresponds to a series of cognitive processes (working memory, inhibitory control, and cognitive flexibility) that are responsible for systematizing behaviours to perform complex tasks [4]. In early childhood, due to the brain immaturity, it is difficult to differentiate cognitive processes. Previous studies have widely assessed the score in Go / No Go test as a measure of EF in this population $[5,6]$. Nonetheless, the importance of reaction time (RT), a measure of processing information speed [7], that is also related to brain white matter development [8], should be considered. In addition, the accuracy of the No Go (Acc-No Go), the child's ability to inhibit continuous behaviour, is also an important measure to be considered, given its rapid growth in early childhood [9], and its relation with the prefrontal cortex maturation [10], the basis of higher order cognitive processes [11].

In young children, SB negatively affects white matter [12], which is related to cognitive processes [13]. However, the effects of SB on EF may vary according to the "sedentary activity" performed [14]. Indeed, activities that promote learning, such as puzzles or thinking games may improve children's EF [14]. Children attending preschools spend a great part of their daily time being sedentary [15]. Nonetheless, 
this time may not necessarily be detrimental to their EF, and whether SB inside and outside preschools is associated to EF outcomes is still unknown.

Movement behaviours are interrelated [16], and physical activity (PA) may mitigate the harmful effects of excess SB [17]. Furthermore, individual characteristics, such as sex [18], age [19] and body mass index (BMI) [20], may have an important role on the relationship between SB and EF. Thus, the present study aimed to analyse the association between SB inside and outside preschools and EF tasks in preschool children. We hypothesized SB inside the preschool is positively associated with EF, while the time spent outside preschools is negatively associates with preschooler's EF.

\section{Methods}

This cross-sectional study used baseline data from the "Movement's Cool" project, aiming to analyze the association between movement behaviours and health outcomes in preschoolers [21]. All the ethical aspects were followed. The evaluation methods and procedures were approved by the Research Ethics Committee of Health Science Center (protocol n. 2.727.698), and by the Education Board of the João Pessoa, Brazil city. Written consents were obtained before participation from the preschools' principals and children's parents.

\subsection{Context and Participants}

Preschool children aged three-to-five-years-old, of both sexes, and registered in Early Education Childhood Centers (EECCs) of João Pessoa / Brazil were eligible. The preschool public education zone is organized in nine districts, where fifty-five EECCs with registered students three-to-five-years-old, are located. Ten EECCs, located in deprived areas of six different districts agreed to participate in the study. For this study, three EECCs, situated in three different districts were conveniently selected. These three preschools were located in deprived areas, with low socioeconomic status (SES): $50.5 \%$ of the mothers or fathers were unemployed, and over $71.8 \%$ of the mothers had not finished high school. The Human Development Index ( $\mathrm{HDI}$ ) form the areas where preschools are located ranges from 0.4 to 0.5 . All parents of registered children (283 children) were invited, and 146 accepted to participate. Nonetheless, 73 children did not validate accelerometer data or did not perform the EF test.

\subsection{Study design}

Measurements were performed during four months (August to October 2019, and March 2020). All the schools and parents were informed about the project's protocols and procedures in meetings with the project coordinator and agreed to participate. All children authorized by their parents were evaluated. A prior meeting with the school's manager was conducted during the first day at schools. On the second day, the socio-demographic data (sex and age) were provided by parents in a face-to-face interview. On the third day, EF data was collected, and the accelerometer was given to each child.

\subsection{Variables and protocols}

\subsubsection{Sedentary behaviour and physical activity}


SB was objectively assessed using accelerometers (Actigraph, model WGT3-X, Florida). The preschool teachers and the parents/guardians received verbal and written instructions for the correct use of the accelerometer. Throughout the week, parents received three phone calls to remind them about the accelerometer use. The device initialization, data reduction and analysis were performed using the ActiLife software (Version 6.13.3).

The participants were instructed to wear the accelerometer on the right hip for 7 consecutive days (Wednesday morning to Tuesday afternoon). Children were allowed to remove the device during waterbased activities and while sleeping (at night). During preschool time, accelerometers were removed by teachers around 11a.m for children's bath and fastened properly after it. Accelerometers were setup to measure acceleration at a $100 \mathrm{~Hz}$ sampling rate, and analyzed as ActiGraph counts, using a 15-s epoch length, and reintegrated to 60 -second epochs for analysis. Periods of $\geq 20 \mathrm{~min}$ of consecutive zero counts were defined as non-wear time and removed from the analysis, and the first day of accelerometer data was omitted from analysis to avoid subject reactivity. Data with a minimum of three days (one weekend and two-week days), with a minimum of $8 \mathrm{~h}$ of wear time was considered valid. The mean wear time was 10.9 hours ( $S D \pm 1.4 \mathrm{~h}$ of wear time between children). The amount of time spent in SB was estimated as $\leq 819$ counts, as proposed by Butte [22]. The time in SB inside of the school was considered from $7 \mathrm{am}$ to $5 \mathrm{pm}$. The time in SB outside of school in the week was recorded from $5 \mathrm{pm}$ until the moment of withdrawal to sleep. On weekends, the SB was considered from the moment the child put the accelerometer in the morning on until withdrawal for night sleep. The Moderate to Vigorous Physical Activity (MVPA), expressed as minute per day, was analyzed as the adjust variable, and was estimated as $>3.908$ counts [22].

\subsubsection{Body Mass Index}

Height $(\mathrm{cm})$ and weight $(\mathrm{kg})$ were determined using a Holtain stadiometer and digitized weighing scale (Seca 708), respectively, while the participant was lightly dressed and barefoot, following a standardized procedure [23]. BMI was calculated by dividing body weight by the squared height in meters $\left(\mathrm{kg} / \mathrm{m}^{2}\right)$.

\subsubsection{Executive function}

EF was assessed using Early Years Toolbox - EYT [24], which is a battery of computerized tasks that was developed to assess the EF of children aged three-to-five-years-old. The battery consists of five tasks assessed from games in an app designed for iPad.

In preschoolers' immature-brain, inhibitory control, working memory, and cognitive flexibility share common processes being challenging to disassociate [25]. Also, it is relevant to consider that in early childhood, these components are strongly related to inhibition, both at the representational level or to the maintenance of objectives [26-28]. Thus, for this study, the accuracy computed in Go, the RT in Go, and the accuracy computed in No Go tasks were considered as outcomes. Children were instructed to tap the screen whenever they saw a fish (Go) and not tap the screen when a shark appeared (No Go). Each stimulus trial remained on the screen for $1500 \mathrm{~ms} 0 \mathrm{~s}$, followed by inter stimulus interval of $1000 \mathrm{~ms} 0 \mathrm{~s}$. Children completed three blocks of 25 trials (a total of 75 trials), with $80 \%$ Go trials (60 fishes) and $20 \%$ 
No Go trials (15 sharks) that were presented in randomized order. Previous studies have shown that the Go / No Go test can activate the entire prefrontal cortex (the brain region considered the basis of support for $E F)$, and it is a more robust task than others to establish EF performance [28, 29].

For analysis, one point was assigned for each correct answer, with the score ranging from 0 to 60 points for Go and 0 to 15 points for No Go. The Inverse Efficiency (IE), computed by the ratio between Reaction time and Accuracy of Go was calculated. This measure has recently been used in EF performance surveys to consider the trade-offs between speed-accuracy [30]. The underlying assumption is that "differences in reaction time performance would decrease if differences in accuracy are large, but would remain the same if accuracy is identical" [31]. The task of Go it is considered a measure of selection attention and speed of response selection [7, 32]. Besides, the accuracy of No Go (Ac-No Go), which is related to inhibition processes, was used for analysis. This protocol presents satisfactory reliability values with Cronbrach's $a=0.95$ [24]. In the current study, the composite reliability values for Go/No-Go was 0.78 , which is considered an adequate value [33].

\subsection{Data Analysis}

To characterize the sample, mean and standard deviation were used, and the difference between sex was verified by Student's T-test. To assess possible associations between EF and SB, with sex, age, BMI and MVPA as covariates, a structural equation model, using multivariate linear regression analysis, was conducted. The presence of univariate and multivariate outliers was verified by the square distance of Mahalanobis $\left(\mathrm{D}^{2}\right)$. Variable's normality was assessed by the coefficients of asymmetry (sk) and uni and multivariate kurtosis (ku). Possible multicollinearities were investigated using VIF (Variance Inflation Factor), and VIF $>5$ was considered a multicollinearity indicator [34]. The robust maximum likelihood method was used [35]. The Mplus program (version 8.0) was used, and values of $p<0.05$ were considered significant.

\section{Results}

A total of 73 preschoolers who completed the entire assessment's protocol participated in the study. Girls showed longer time in SB within EECC compared to boys (Table 1). 
Table 1

Sample's characteristics.

\begin{tabular}{|c|c|c|c|c|c|c|}
\hline \multirow[t]{2}{*}{ Variables } & \multicolumn{2}{|c|}{ Boys $(n=29)$} & \multicolumn{2}{|c|}{ Girls $(n=44)$} & \multirow[t]{2}{*}{ p } & \multirow[t]{2}{*}{ Cohen's d } \\
\hline & Mean & SD & Mean & SD & & \\
\hline Age (months) & 54.3 & 8.9 & 55.5 & 9.2 & 0.612 & -0.122 \\
\hline $\mathrm{BMI}\left(\mathrm{kg} / \mathrm{m}^{2}\right)$ & 15.1 & 1.3 & 15.6 & 2.1 & 0.234 & -0.287 \\
\hline MVPA (min/day) & 65.2 & 23.0 & 69.5 & 21.2 & 0.413 & -0.197 \\
\hline SB-inside (min/day) & 233.7 & 63.1 & 266.2 & 69.9 & 0.047 & -0.482 \\
\hline SB-outside (min/day) & 182.0 & 70.6 & 192.8 & 69.4 & 0.522 & -0.154 \\
\hline SB-wkn (min/day) & 442.9 & 158.2 & 452.0 & 168.6 & 0.817 & -0.055 \\
\hline SB (min/day) & 858.6 & 252.3 & 911.0 & 257.0 & 0.394 & -0.205 \\
\hline Ac-Go (score) & 50.9 & 6.3 & 49.4 & 8.6 & 0.433 & 0.189 \\
\hline RT-Go (ms) & 290 & 30 & 300 & 30 & 0.196 & -0.312 \\
\hline IE (RT/Ac) & 5.8 & 1.2 & 6.5 & 3.0 & 0.253 & -0.277 \\
\hline Ac-No Go (score) & 10.4 & 3.5 & 11.6 & 2.0 & 0.067 & -0.445 \\
\hline \multicolumn{7}{|c|}{$\begin{array}{l}\text { SB-inside = Sedentary behaviour in weekdays inside; SB-outside = Sedentary behaviour in weekdays } \\
\text { outside; SB-wkn = Sedentary behaviour in weekend days; } \mathrm{SB}=\text { Sedentary behaviour all day; Ac-Go = } \\
\text { Accuracy of Go; RT-Go = Reaction time in Go; Ac-No Go = Accuracy of No Go; Statistically significant } \\
\text { values are shown in bold }(\mathrm{p}<0.05) \text {. }\end{array}$} \\
\hline
\end{tabular}

Analysing the associations between SB (i.e. SB-inside, SB-outside and SB-wkn), and EF (i.e., IE, and Ac-No Go), adjusted for sex, age, BMI and MVPA (Fig. 1), a significant and positive association between SB-wkn and IE of Go $(b=0.61 ; p<0.001)$ was observed. The model's general explaining $51.9 \%$ of IE, and $2 \%$ of Ac-No Go.

\section{Discussion}

The present study analysed the association between time spent in SB in different contexts and EF in preschoolers. Although prior studies have assessed the relation between SB and EF in preschoolers [36, 37], the current study adds important findings to the existing literature, considering distinctive contexts where preschoolers are sedentary.

The study's primary results showed that time spent in SB on weekend days was positively related to children's IE, a measure to consider the trade-offs between speed-accuracy [30]. Indeed, a lower IE is associated to a more efficient task performance [30], what might indicate a harmful for children's IE. Thus, the excessive time spent being sedentary during weekend seems to be detrimental to preschoolers' 
EF [14]. According to Lillard and colleagues [38], the information processing follows an inverted U shape, in such a way that the excess of information, as well as the content children are exposed, may lead to children's EF impairments [39]. Moreover, the assessed children spend twice as much time on SB during weekends than on weekdays. Excessive SB at this stage of life is associated with decreased white matter integrity in brain regions that support EF, such as the frontal and the prefrontal Thalamus [12].

It is important to highlight that the relationship between SB and EF in preschoolers is controversial [14, 40], partly due to the type of SB performed, and to the time spent being sedentary. The technology advances, and the easier access to electronic devices may predispose early age children to a greater amount of time exposed to screens [41], though specific educational TV, tablet, and computer programs developed for this public may positively contribute to children's learning process [42].

Although it is expected that sedentary time inside preschools could positively predict children's EF, the current study showed no significant association with any of the assessed EF outcomes. Preschool's context characteristics, such as inadequate material, lack of space, and teachers qualification to work with these specific age group, may influence the quality of the offered classes, and consequently, children 's cognitive learning [43]. The assessed children spend 10 hours per day at public preschools, located in low-income areas of a middle-income country, where social aspects that directly interfere with child's development cannot be ignored. In addition, in several cases, parents are involved in an extensive work routine, that may contribute to a lack of quality interactions with their children, especially during week days, and consequently, on children's cognitive development [44].

The main strength of the current study is the analysis of sedentary time in different contexts and its repercussion in preschoolers' EF. As far as we know, this is the first study to report such associations in young children, considering the context where SB happens, what may provide a new insight in the research field. Besides, the studied sample covers a critical geographical gap in this research area. However, our findings should be considered with certain parsimony, as the lack of information regarding the type/quality of SB performed in each context may be determinant to the reported associations. Finally, other factors involved with preschoolers' EF (e.g., home and school environments, diet, motor skills, self-esteem, brain-derived factors, and others) [45] could be detrimental for the fit indexes, and should be further explored. Therefore, these outcomes emphasize a context-dependent influence of SB time on preschoolers' $E F$, and demonstrate the relevance and priority of further studies to better discern types of SB.

\section{Conclusion}

The model presented in this study showed that SB on weekend days showed a significant and adverse association with children's IE, suggesting that excessive SB on these days may be harmful to EF in early child. Therefore, our findings suggest that investigating the different contexts where preschoolers are engaged in SB may be decisive for ensuring enhanced children's EF profile, although strategies focusing in reducing SB in early stages of life should be a priority. 


\section{List Of Abbreviations}

$\mathbf{A C}=$ accuracy

$\mathrm{BMI}=$ body mass index

EF = executive function

EECC $=$ Early Education Childhood Centers

IE = inverse efficiency

MVPA $=$ moderate to vigorous physical activity

SB = sedentary behaviour

\section{Declarations}

Funding: N/A

Conflicts of interest/Competing interests: N/A

Availability of data and material: N/A

Code availability: N/A

Authors' contributions: Substantial contributions to conception and design, acquisition of data, or analysis and interpretation of data: Bezerra, Esteban-Cornejo, Goulart, Bandeira, Souza Filho. Drafting the article or revising it critically for important intellectual content: All the authors. Final approval of the version to be published: Mota and Martins.

Ethics approval: All procedures were approved by the university committee and the board of education. The Helsinki Declarations' ethical aspects were followed (Association, 2013). The Research Ethics Committee of the Health Science Center of the Federal University of Paraiba and the local board of education approved the study (protocol n. 4.102.806).

Consent to participate: All the preschools' staff and parents were informed about the research's goals, protocols, and procedures in meetings with the project coordinator (one session in each school) and agreed to participate in the present study through an informed consent form.

Consent for publication: All preschool staff and parents were informed about the research objectives, protocols, and procedures in meetings with the project coordinator (one session at each school) and agreed to publish the data, maintaining the confidentiality of the participants, through an informed consent form. 


\section{References}

1. Young Deborah, R., et al., Sedentary Behavior and Cardiovascular Morbidity and Mortality: A Science Advisory From the American Heart Association. Circulation, 2016. 134(13): p. e262-e279.

2. Rodriguez-Ayllon, M., et al., Role of Physical Activity and Sedentary Behavior in the Mental Health of Preschoolers, Children and Adolescents: A Systematic Review and Meta-Analysis. Sports Medicine, 2019. 49(9): p. 1383-1410.

3. Zavala-Crichton, J.P., et al., Association of Sedentary Behavior with Brain Structure and Intelligence in Children with Overweight or Obesity: The ActiveBrains Project. Journal of clinical medicine, 2020. 9(4): p. 1101.

4. Bell, M.A. and K. Cuevas, Psychobiology of executive function in early development. 2016. p. 157179.

5. Wiebe, S.A., T.D. Sheffield, and K.A. Espy, Separating the Fish From the Sharks: A Longitudinal Study of Preschool Response Inhibition. Child Development, 2012. 83(4): p. 1245-1261.

6. Bezerra, T.A., et al., 24-hour movement behaviour and executive function in preschoolers: $A$ compositional and isotemporal reallocation analysis. European Journal of Sport Science, 2020: p. 19.

7. Anderson, M., T. Nettelbeck, and J. Barlow, Reaction time measures of speed of processing: Speed of response selection increases with age but speed of stimulus categorization does not. British Journal of Developmental Psychology, 1997. 15(2): p. 145-157.

8. Scantlebury, N., et al., Relations between White Matter Maturation and Reaction Time in Childhood. Journal of the International Neuropsychological Society, 2014. 20(1): p. 99-112.

9. Carver, A.C., D.J. Livesey, and M. Charles, Age Related Changes in Inhibitory Control as Measured by Stop Signal Task Performance. International Journal of Neuroscience, 2001. 107(1-2): p. 43-61.

10. Tamm, L., V. Menon, and A.L. Reiss, Maturation of Brain Function Associated With Response Inhibition. Journal of the American Academy of Child \& Adolescent Psychiatry, 2002. 41(10): p. 12311238.

11. Fuster, J., The prefrontal cortex. 2015: Academic Press.

12. Hutton, J.S., et al., Associations Between Screen-Based Media Use and Brain White Matter Integrity in Preschool-Aged Children. JAMA Pediatrics, 2020. 174(1): p. e193869-e193869.

13. Dodson, C.K., et al., White matter properties associated with pre-reading skills in 6-year-old children born preterm and at term. Developmental medicine and child neurology, 2018. 60(7): p. 695-702.

14. Carson, V., et al., Systematic review of sedentary behavior and cognitive development in early childhood. Preventive Medicine, 2015. 78: p. 115-122.

15. Barbosa, S.C., et al., School environment, sedentary behavior and physical activity in preschool children. Revista paulista de pediatria, 2016. 34(1984-0462 (Electronic)): p. 8.

16. Pearson, N., et al., Associations between sedentary behaviour and physical activity in children and adolescents: a meta-analysis. Obesity reviews : an official journal of the International Association for 
the Study of Obesity, 2014. 15(8): p. 666-675.

17. Ekelund, U., et al., Does physical activity attenuate, or even eliminate, the detrimental association of sitting time with mortality? A harmonised meta-analysis of data from more than 1 million men and women. The Lancet, 2016. 388(10051): p. 1302-1310.

18. Tanaka, C., M. Tanaka, and S. Tanaka, Objectively evaluated physical activity and sedentary time in primary school children by gender, grade and types of physical education lessons. BMC public health, 2018. 18(1): p. 948-948.

19. Júdice, P.B., et al., Sedentary patterns, physical activity and health-related physical fitness in youth: a cross-sectional study. The international journal of behavioral nutrition and physical activity, 2017. 14(1): p. 25-25.

20. Biddle, S.J.H., E. García Bengoechea, and G. Wiesner, Sedentary behaviour and adiposity in youth: a systematic review of reviews and analysis of causality. The international journal of behavioral nutrition and physical activity, 2017. 14(1): p. 43-43.

21. Martins, C.M.d.L., et al., Adherence to 24-hour movement guidelines in low-income Brazilian preschoolers and associations with demographic correlates. American Journal of Human Biology, 2020. $\mathbf{n} / \mathbf{a}(\mathrm{n} / \mathrm{a})$ : p. e23519.

22. Butte, N., et al., Prediction of Energy Expenditure and Physical Activity in Preschoolers. Medicine and science in sports and exercise, 2013. 46.

23. de Onis, M., WHO child growth standards, W.H. Organization, Editor. 2006, World Health Organization: Geneva.

24. Howard, S. and E. Melhuish, An Early Years Toolbox for Assessing Early Executive Function, Language, Self-Regulation, and Social Development: Validity, Reliability, and Preliminary Norms. Journal of Psychoeducational Assessment, 2016. 10.

25. Diamond, A., Executive Functions. Annual review of psychology, 2012. 64.

26. Miyake, A., et al., The Unity and Diversity of Executive Functions and Their Contributions to Complex "Frontal Lobe" Tasks: A Latent Variable Analysis. Cognitive psychology, 2000. 41: p. 49-100.

27. Karr, J.E., et al., The unity and diversity of executive functions: A systematic review and re-analysis of latent variable studies. Psychol. Bull, 2018. 144(1939-1455 (Electronic)): p. 38.

28. Willoughby, M.T., et al., The measurement of executive function at age 5: psychometric properties and relationship to academic achievement. Psychol. Assess., 2012. 24(1939-134X (Electronic)): p. 13.

29. Smith, E., et al., Prefrontal Activation During Executive Tasks Emerges Over Early Childhood: Evidence From Functional Near Infrared Spectroscopy. Developmental Neuropsychology, 2017. 42(4): p. 253264.

30. Suchodoletz, A., P.L. Slot, and D.M. Shroff, Measuring executive function in Indian mothers and their 4-year-old daughters. PsyCh Journal, 2017. 6(1): p. 16-28. 
31. Ding, X.P., et al., Elementary school children's cheating behavior and its cognitive correlates. Journal of experimental child psychology, 2014. 121: p. 85-95.

32. Willner, C.J., Gatzke-Kopp, L. M., Bierman, K. L., Greenberg, M. T., \& Segalowitz, S. J, Relevance of a neurophysiological marker of attention allocation for children's learning-related behaviors and academic performance. Developmental Psychology, 2015. 51(8): p. 1148-1162.

33. Valentini, F. and B.F. Damásio, Variância Média Extraída e Confiabilidade Composta: Indicadores de Precisão. Psicologia: Teoria e Pesquisa, 2016. 32.

34. Marôco, J., Análise de equações estruturais: Fundamentos teóricos, software \& aplicações. 2010: ReportNumber, Lda.

35. Byrne, B.M., Structural equation modeling with LISREL, PRELIS, and SIMPLIS: Basic concepts, applications, and programming. 2013, New York: Psychology Press.

36. Schmidt, M.E., et al., Television Viewing in Infancy and Child Cognition at 3 Years of Age in a US Cohort. Pediatrics, 2009. 123(3): p. e370.

37. Nathanson, A., et al., The Relation Between Television Exposure and Executive Function Among Preschoolers. Developmental psychology, 2014. 50.

38. Lillard, A.S., H. Li, and K. Boguszewski, Chapter Seven - Television and Children's Executive Function, in Advances in Child Development and Behavior, J.B. Benson, Editor. 2015, JAl. p. 219-248.

39. Lillard, A. and J. Peterson, The Immediate Impact of Different Types of Television on Young Children's Executive Function. Pediatrics, 2011. 128: p. 644-9.

40. Cerniglia, L. and S. Cimino, A Reflection on Controversial Literature on Screen Time and Educational Apps Use in 0-5 Years Old Children. International Journal of Environmental Research and Public Health, 2020. 17.

41. Rideout, V., Zero to eight: children's media use in America. San Francisco: Common Sense Media, viewed 03 Dec. 2011. 2016.

42. Plowman, L., et al., Preschool children's learning with technology at home. Computers \& Education, 2012. 59(1): p. 30-37.

43. Burchinal, M.R., et al., Relating Quality of Center-Based Child Care to Early Cognitive and Language Development Longitudinally. Child Development, 2000. 71(2): p. 339-357.

44. John, A., K. Finch, and A. Tarullo, Socioeconomic status and neural processing of a go/no-go task in preschoolers: An assessment of the P3b. Developmental Cognitive Neuroscience, 2019. 38: p. 100677.

45. Takeuchi, H. and R. Kawashima, Neural Mechanisms and Children's Intellectual Development: Multiple Impacts of Environmental Factors. The Neuroscientist : a review journal bringing neurobiology, neurology and psychiatry, 2015. 22.

\section{Figures}




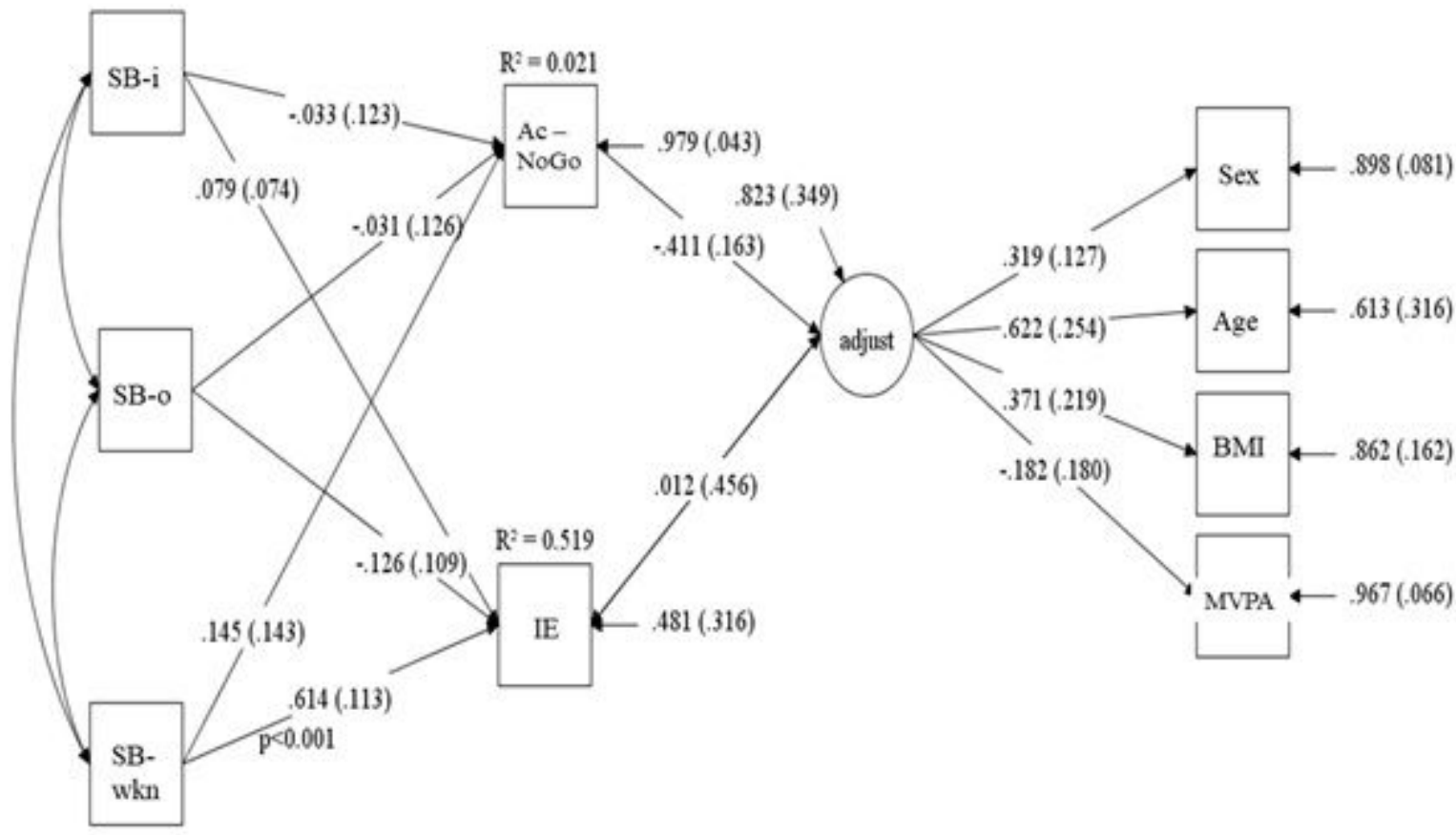

\section{Figure 1}

Associations between SB and EF. Legend: SB-i = Sedentary behaviour in weekdays inside; $\mathrm{SB}-\mathrm{o}=$ Sedentary behaviour in weekdays outside; SB-wkn = Sedentary behaviour in weekend days; Ac = Accuracy; IE = Inverse Efficiency; BMI = Body Mass Index; MVPA = Moderate to Vigorous Physical Activity. 